\title{
Vitamin $D$ deficiency is associated with the severity of COPD: a systematic review and meta-analysis
}

\author{
Biyuan Zhul,* \\ Biqing Zhu',* \\ Chaolie $\mathrm{Xiao}^{3}$ \\ Zhiwen Zheng'
}

'The Fifth Internal Medicine Department, ${ }^{2}$ Department of Laboratory Medicine, ${ }^{3}$ Department of Intensive Care Unit, Dongguan Hospital of Traditional Chinese Medicine, Guangzhou University of Chinese Medicine, Dongguan City, People's Republic of China

*These authors contributed equally to this work
Correspondence: Zhiwen Zheng The Fifth Internal Medicine Department, Dongguan Hospital of Traditional Chinese Medicine, Guangzhou University of Chinese Medicine, Songshan Lake Avenue 22, Dongcheng District, Dongguan City, Guangdong Province 523000, People's Republic of China

Tel +8676926388923

Fax +8676926388923

Email zhengzhiwen20I4@I63.com
This article was published in the following Dove Press journal:

International Journal of COPD

II September 2015

Number of times this article has been viewed

Purpose: To explore the association between host serum 25-hydroxyvitamin D (25(OH)D) and the susceptibility and severity of COPD.

Methods: Previous studies on the association between host $25(\mathrm{OH}) \mathrm{D}$ and the susceptibility and severity of COPD were collected on the basis of a systematic literature search of PubMed and Web of Science up to June 2015. Continuous variable data were presented as standard mean difference (SMD) or weighted mean difference with $95 \%$ confidence interval (CI). The dichotomous variable data were analyzed as relative ratio (RR) or odds ratio with $95 \%$ CI for cohort and case-control studies. A systematic review was conducted to understand the curative and side effects of vitamin D intake.

Results: A total of 18 studies including eight cohort, five case-control, and five randomized studies met the inclusion criteria. The serum level of 25(OH)D in COPD patients was comparable with controls with a pooled SMD of $0.191(95 \% \mathrm{CI}:-0.126$ to $0.508, P=0.237)$ based on pooled analyses of cohort studies. However, the serum level of $25(\mathrm{OH}) \mathrm{D}$ in COPD patients was lower with a pooled SMD of 0.961 (95\% CI: 0.476-1.446, $P<0.001)$ compared with controls based on pooled analyses of case-control studies. The deficiency rates of $25(\mathrm{OH}) \mathrm{D}$ were comparable between controls and COPD patients with a pooled RR of 0.955 ( $95 \%$ CI: $0.754-1.211, P=0.705)$ based on analyses of cohort studies, and the same results were observed based on pooled analyses of case-control studies. Interestingly, the deficiency rate of $25(\mathrm{OH}) \mathrm{D}$ was significantly lower in moderate or severe COPD patients with a pooled RR of 0.723 (95\% CI: $0.632-0.828, P<0.001$ ) compared with that in mild COPD patients. The same results were obtained from the pooled analysis between moderate and severe COPD patients. The four randomized studies showed that vitamin D intake provided benefit for COPD patients.

Conclusion: Low serum levels of $25(\mathrm{OH}) \mathrm{D}$ were not associated with COPD susceptibility, but the high deficiency rate of $25(\mathrm{OH}) \mathrm{D}$ was associated with COPD severity. Vitamin D supplementation may prevent COPD exacerbation.

Keywords: $25(\mathrm{OH}) \mathrm{D}, \mathrm{COPD}$, susceptibility

\section{Introduction}

COPD, a systemic inflammatory disease, is characterized by airflow limitation that is not fully reversible. ${ }^{1}$ It has been estimated that the average prevalence of COPD is $6.2 \%$ in Asia and almost one-fifth of identified subjects are categorized as having severe COPD. ${ }^{2}$ COPD is a major cause of chronic morbidity and mortality ${ }^{3}$ and is currently the fourth leading cause of death. The World Health Organization predicts that COPD will become the third leading cause of death worldwide by $2020 .{ }^{3,4}$ Due to multiple factors involved in the pathogenesis of COPD, the patterns of mechanisms and progression of COPD are extremely difficult to dissect. While it has been widely accepted that tobacco 
smoking is a major risk factor, a small number of long-term smokers develop symptomatic airflow obstruction. ${ }^{5}$ Thus, lots of other factors might also play important roles in the morbidity and progression of COPD.

It has been reported that vitamin $\mathrm{D}$ is associated with bone health by affecting calcium homeostasis. ${ }^{6,7}$ However, some studies have also suggested that vitamin D is associated with the pathogenesis of a number of autoimmune diseases ${ }^{8,9}$ as well as the development of cancer, ${ }^{10}$ asthma, ${ }^{11}$ and infections including respiratory infection ${ }^{12}$ and tuberculosis. ${ }^{13}$ Vitamin $\mathrm{D}$ may be involved in the pathogenesis and affect the severity of COPD in several ways, such as reducing the frequency of respiratory infections, impairing response to pathogens, and inhibiting the proliferation of airway smooth muscle. ${ }^{14}$ The main carrier of vitamin D metabolites is VDBP. The polymorphisms of VDBP gene have been associated with COPD and the serum level of 25-hydroxyvitamin D $(25(\mathrm{OH}) \mathrm{D}) .{ }^{15,16}$ However, it has been reported that low levels of $25(\mathrm{OH}) \mathrm{D}$ was not consistent with vitamin D deficiency. ${ }^{17}$ Two cohort studies suggested that vitamin D was not associated with COPD based on the observation that the serum levels of $25(\mathrm{OH}) \mathrm{D}$ were comparable between healthy subjects and COPD patients. ${ }^{18,19}$ However, this conclusion was questioned by other studies..$^{20,21}$

In the present study, we conducted a meta-analysis to evaluate the association between the serum level of $25(\mathrm{OH}) \mathrm{D}$ and the susceptibility and severity of COPD. We focused on four aspects: 1) the serum level of $25(\mathrm{OH}) \mathrm{D}$ between controls and COPD patients; 2$)$ the deficiency rates of 25(OH)D between controls and COPD patients; 3$)$ the deficiency rates of $25(\mathrm{OH}) \mathrm{D}$ in mild, moderate, and severe COPD patients; and 4) the curative effect of supplying vitamin D for COPD patients.

\section{Materials and methods Literature retrieval}

Computerized searches in the National Center for Biotechnology Information PubMed and Web of Science were conducted by two authors (Biyuan Zhu and Biqing Zhu). The search time window was up to June 2015 and all articles searched for were in English. The following keywords were used for literature searching: "COPD" or "chronic obstructive pulmonary disease" combined with "vitamin d", "vit d", "cholecalciferol", or "hydroxyvitamin. We also manually searched the reference lists of the retrieved articles to identify additional qualified studies.

\section{Definitions}

COPD patients exhibited stable COPD and acute exacerbation COPD. The severity of COPD was defined on the basis of percentage of the forced expiratory volume in 1 second predicted according to the Global Initiative for Chronic Obstructive Lung Disease (GOLD) criteria., ${ }^{3,22-25}$ The controls were healthy or patients without COPD. Patients with plasma level of $25(\mathrm{OH}) \mathrm{D}$ less than $20 \mathrm{ng} / \mathrm{mL}(50 \mathrm{nmol} / \mathrm{L})$ were categorized into the $25(\mathrm{OH}) \mathrm{D}$ deficiency group. ${ }^{6,20}$

\section{Inclusion and exclusion criteria}

Studies were included into the present meta-analysis based on the following criteria. To analyze the serum level of $25(\mathrm{OH}) \mathrm{D}$ in controls and COPD patients: 1) the study reported the serum level of $25(\mathrm{OH}) \mathrm{D}$ with patient number, mean, and standard deviation and 2) the study included controls (healthy or patients without COPD). To investigate the deficiency rates of $25(\mathrm{OH}) \mathrm{D}$ in controls and COPD patients, the study reported the deficiency rate of $25(\mathrm{OH}) \mathrm{D}$ in COPD patients and controls. To study the deficiency rates of $25(\mathrm{OH})$ $\mathrm{D}$ in mild, moderate, and severe COPD patients, the study reported the deficiency rates of $25(\mathrm{OH}) \mathrm{D}$ in $\mathrm{COPD}$ patients and included the severity of COPD (mild, moderate, and severe). To analyze the curative effects of supplying vitamin D for COPD patients: 1) randomized clinical trials were included; and 2) the curative and side effects of vitamin D intake were analyzed. Studies of reviews, in vitro and animal experiments, and comments were excluded from the present meta-analysis.

\section{Quality assessment}

The quality of the observational studies was independently assessed by two authors (Biyuan Zhu and Chaolie Xiao) using the Newcastle-Ottawa Scale (NOS). ${ }^{26}$ Three parameters of quality, including selection, comparability, and exposure/outcome assessments were used in NOS. Studies of low, intermediate, and high-quality studies were defined as NOS scores of 1-3, 4-6, and 7-9, respectively. ${ }^{27}$ The quality of randomized studies was assessed using the Jadad score ${ }^{28}$ that was rated on a scale of 0-5 according to the presence of three features: randomization, blinding, and patient accountability. A higher-quality trial was defined as a study with a Jadad score $\geq 3$. Discrepancies were resolved by consensus after joint reevaluation of the original studies by a third author (Zhiwen Zheng).

\section{Data extraction}

Data were independently extracted by two reviewers (Biyuan Zhu and Chaolie Xiao) and validated by a third reviewer (Zhiwen Zheng). For each eligible study, we extracted first author names, publication year, country, study design, numbers of patients with COPD and controls, and the serum level of $25(\mathrm{OH}) \mathrm{D}$ (mean \pm standard deviation). 


\section{Statistical analyses}

Meta-analysis was performed in STATA 11.0 (StataCorp LP, College Station, TX, USA). Continuous variable data (controls versus COPD patients) were presented as standard mean difference (SMD) or weighted mean difference with $95 \%$ confidence interval (CI). Dichotomous variable data (controls versus COPD patients, mild versus moderate/severe COPD patients, and moderate versus severe COPD patients) were presented as relative ratio (RR) or odds ratio (OR) with $95 \% \mathrm{CI}$ for cohort and case-control studies. In the forest plots, the pooled SMD or weighted mean difference $>0$ represented the higher level of $25(\mathrm{OH}) \mathrm{D}$ in controls, and $\mathrm{RR}$ or $\mathrm{OR}<1$ represented the lower deficiency rates in controls, mild COPD patients, and severe COPD patients, respectively. A chi-squared test was used to assess the heterogeneity. $I^{2}$ value $<25 \%$ was regarded as no heterogeneity. Fixed effects model was used when no significant heterogeneity was identified among the studies analyzed. Random-effects model was performed when significant heterogeneity was identified among the studies analyzed. The publication bias was also evaluated. A $P$-value $<0.05$ was considered to be significant on two-sides.

\section{Results \\ Study search}

Based on the aforementioned search strategies, a total of 450 articles were collected. After removal of review articles $(n=99)$, studies not related with the topic $(n=196)$, commentary articles $(n=30)$, and others $(n=94), 31$ studies were identified for further evaluation. In addition, 13 studies without controls $(n=2)$, lacking essential information $(n=9)$, or without full text $(n=2)$ were excluded. Finally, 18 studies that met the inclusion criteria were included in the present meta-analysis. A flowchart of the search and selection process is shown in Figure 1.

Nine studies containing 919 controls and 990 COPD patients were included to analyze the serum levels of 25(OH)D between controls and COPD patients (Table 1). Among these nine studies, five were case-control studies ${ }^{29-33}$ and four were cohort studies. ${ }^{18-20,34}$ Five studies were of high quality because their NOS scores were not less than 7. ${ }^{19,20,29,31,34}$ Six studies containing 6,375 controls and 2,504 COPD patients were included to analyze the deficiency rates of 25(OH)D in controls and COPD patients (Table 2). Among these six studies, two were case-control studies ${ }^{30,33}$ and four were cohort studies. ${ }^{19-21,34}$ Five studies were of high quality because their NOS scores were not less than 7. ${ }^{19,20,29,31,34}$ Five cohort studies were retrieved to analyze the deficiency rates of 25(OH)D in mild, moderate, and severe COPD patients (Table 2). Among the five studies, three studies ${ }^{20,21,35}$ contained 1,026 mild and 1,298 moderate/severe COPD patients and four studies ${ }^{20,35-37}$ contained 435 moderate and 494 severe COPD patients. Three studies with NOS scores not less than

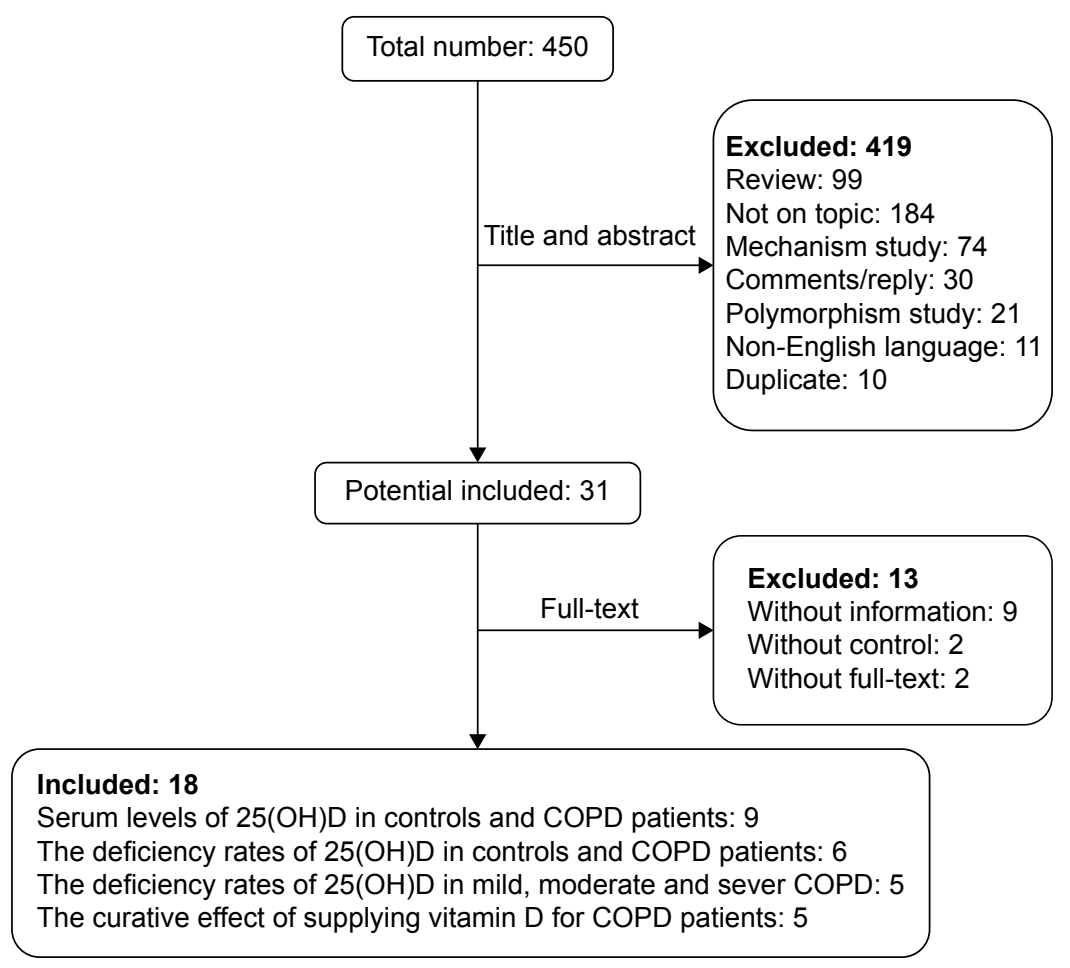

Figure I A flowchart of identified studies.

Abbreviation: 25(OH)D, 25-hydroxyvitamin D.

International Journal of COPD 2015:10

submit your manuscript | www.dovepress.com 

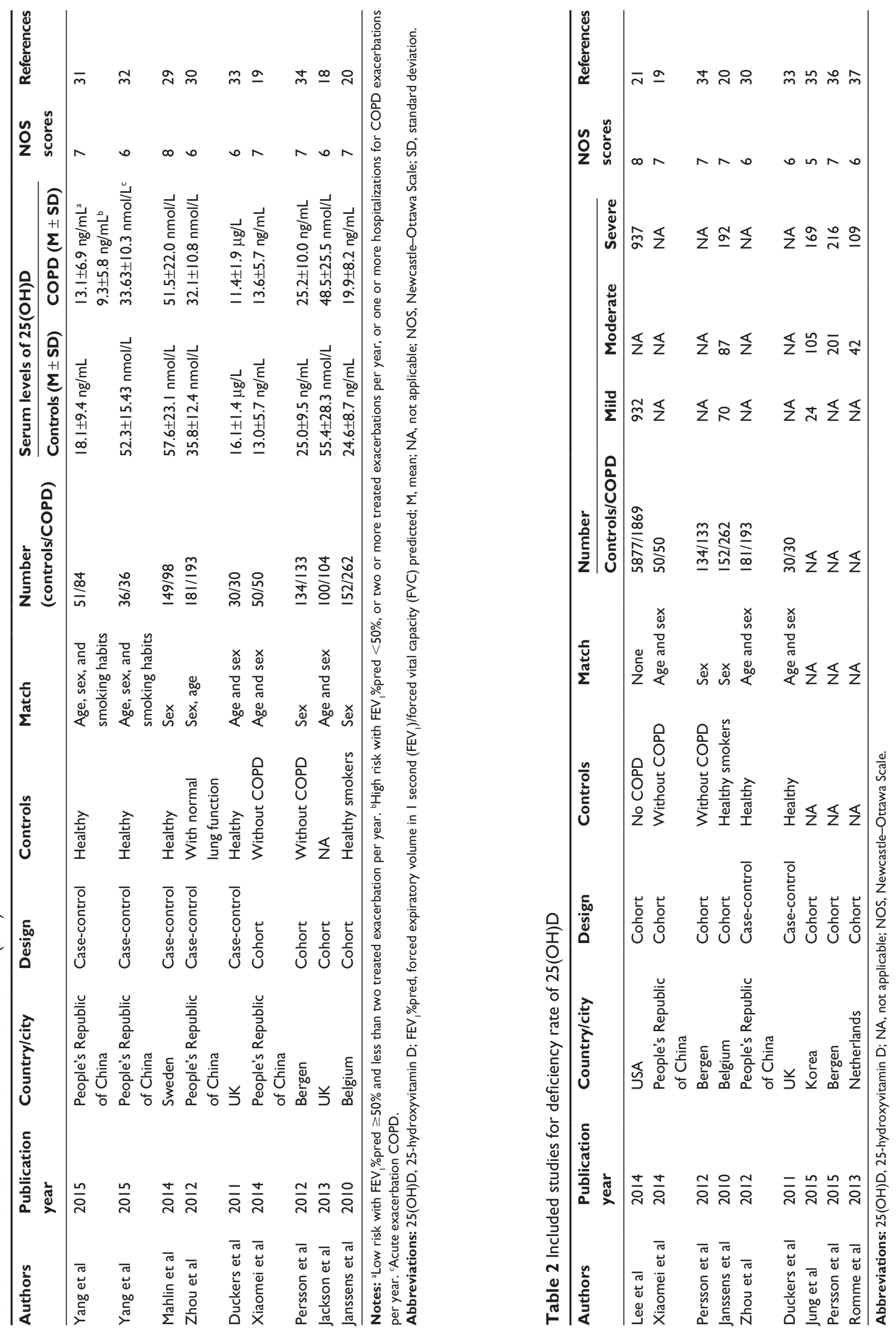
7 were considered as studies of high quality. ${ }^{20,21,36}$ Five randomized studies, ${ }^{38-41}$ containing 296 patients with placebo and 300 patients with vitamin $\mathrm{D}$, were included to analyze the curative effect of supplying vitamin $\mathrm{D}$ for COPD patients (Table 3). According to Jada scores, one study with a score of 1 was considered as a low-quality study. ${ }^{40}$

\section{Meta-analysis of the serum level of $25(\mathrm{OH}) \mathrm{D}$}

A random-effects model for meta-analysis was used to compare the serum level of $25(\mathrm{OH}) \mathrm{D}$ between controls and COPD patients. The results suggested that the serum level of $25(\mathrm{OH}) \mathrm{D}$ in COPD patients was significantly lower than that in controls based on a pooled SMD of 0.596 (95\% CI: $0.305-0.886, P<0.001$ ) compared with controls (Figure 2 ). A significant heterogeneity with $I^{2}=88.5, P<0.001$, and a publication bias were identified based on the Egger's test of $0.372(P=0.046)$.

According to the study design, the results of sensitivity analysis were different. The serum level of $25(\mathrm{OH}) \mathrm{D}$ in COPD patients was comparable with controls with a pooled SMD of 0.191 ( $95 \% \mathrm{CI}:-0.126$ to $0.508, P=0.237)$ based on pooled analyses of cohort studies (Figure 2). No publication bias was identified based on the Egger's test of $0.235(P=0.322)$. However, the serum level of $25(\mathrm{OH}) \mathrm{D}$ in COPD patients was lower than that in controls with a pooled SMD of 0.961 (95\% CI: 0.476-1.446, $P<0.001)$ based on pooled analyses of case-control studies (Figure 2). A publication bias was identified based on the Egger's test of $0.085(P=0.006)$.

\section{Meta-analysis of the deficiency rates of $25(\mathrm{OH}) \mathrm{D}$}

A random-effects model for meta-analysis was conducted to analyze the deficiency rate of $25(\mathrm{OH}) \mathrm{D}$ in controls and COPD patients. The results suggested that the deficiency rate of $25(\mathrm{OH}) \mathrm{D}$ was comparable between these two groups with a pooled RR of 0.955 (95\% CI: $0.754-1.211, P=0.705)$ based on pooled analyses of cohort studies (Figure $3 \mathrm{~A}$ ). A significant heterogeneity was identified with $I^{2}=72.2, P=0.013$, and this result showed no publication bias based on the Egger's test of $0.216(P=0.296)$. The same result was observed in the pooled analysis of case-control studies. No significant difference in the deficiency rate of $25(\mathrm{OH}) \mathrm{D}$ was identified

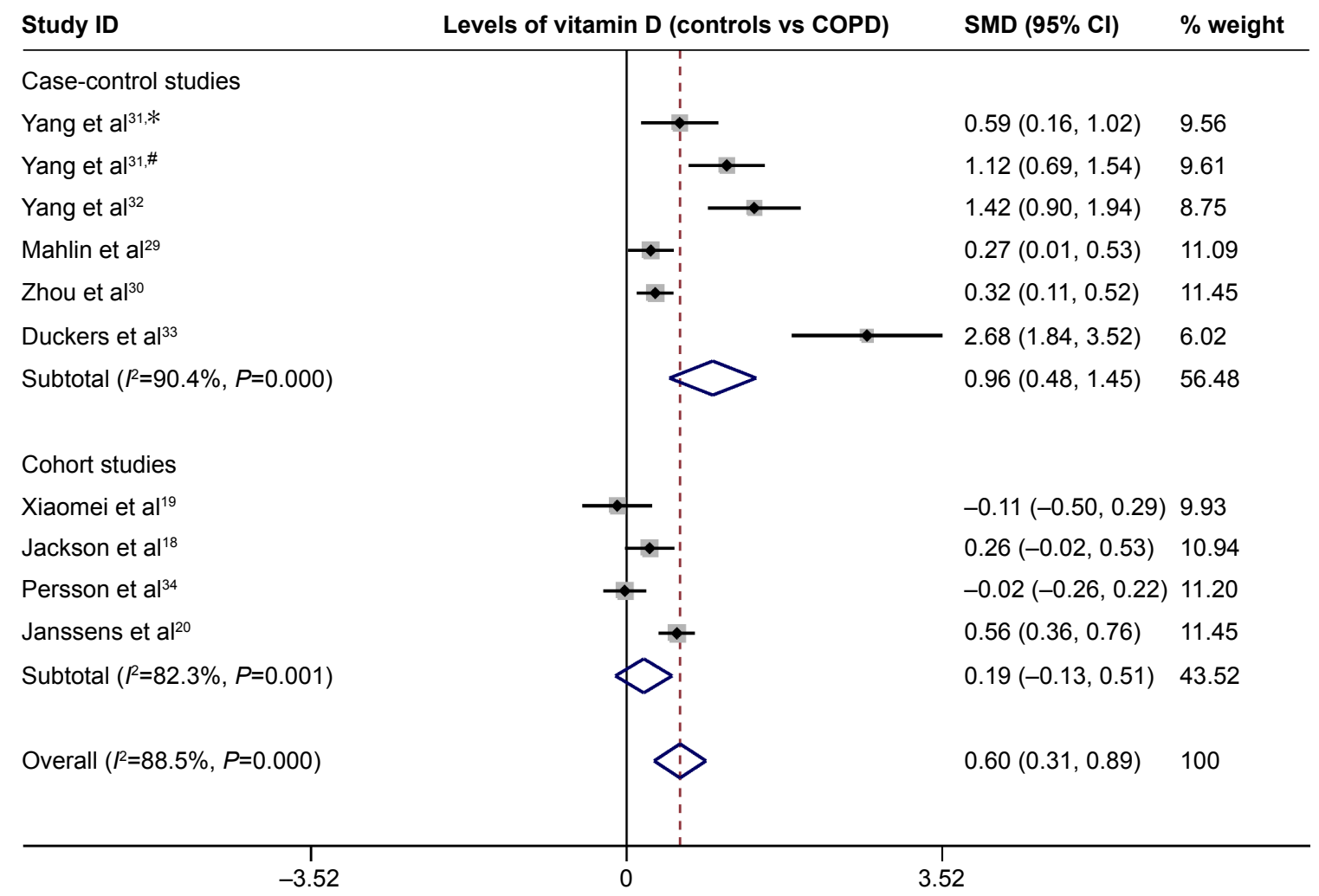

Figure 2 Forest plots of the serum level of $25(\mathrm{OH}) \mathrm{D}$ in the controls and COPD patients.

Notes: Weights are from random-effects analysis. *Low-risk COPD: FEV $\%$ pred $\geq 50 \%$ and less than two treated exacerbation per year. ${ }^{*} \mathrm{High}-$ risk $\mathrm{COPD}$ : FEV $\%$ pred $<50 \%$, or two or more treated exacerbations per year, or one or more hospitalizations for COPD exacerbations per year.

Abbreviations: $\mathrm{Cl}$, confidence interval; $\mathrm{FEV}, \%$ pred, forced expiratory volume in I second $\left(\mathrm{FEV}_{1}\right) /$ forced vital capacity (FVC) predicted; SMD, standard mean difference. 


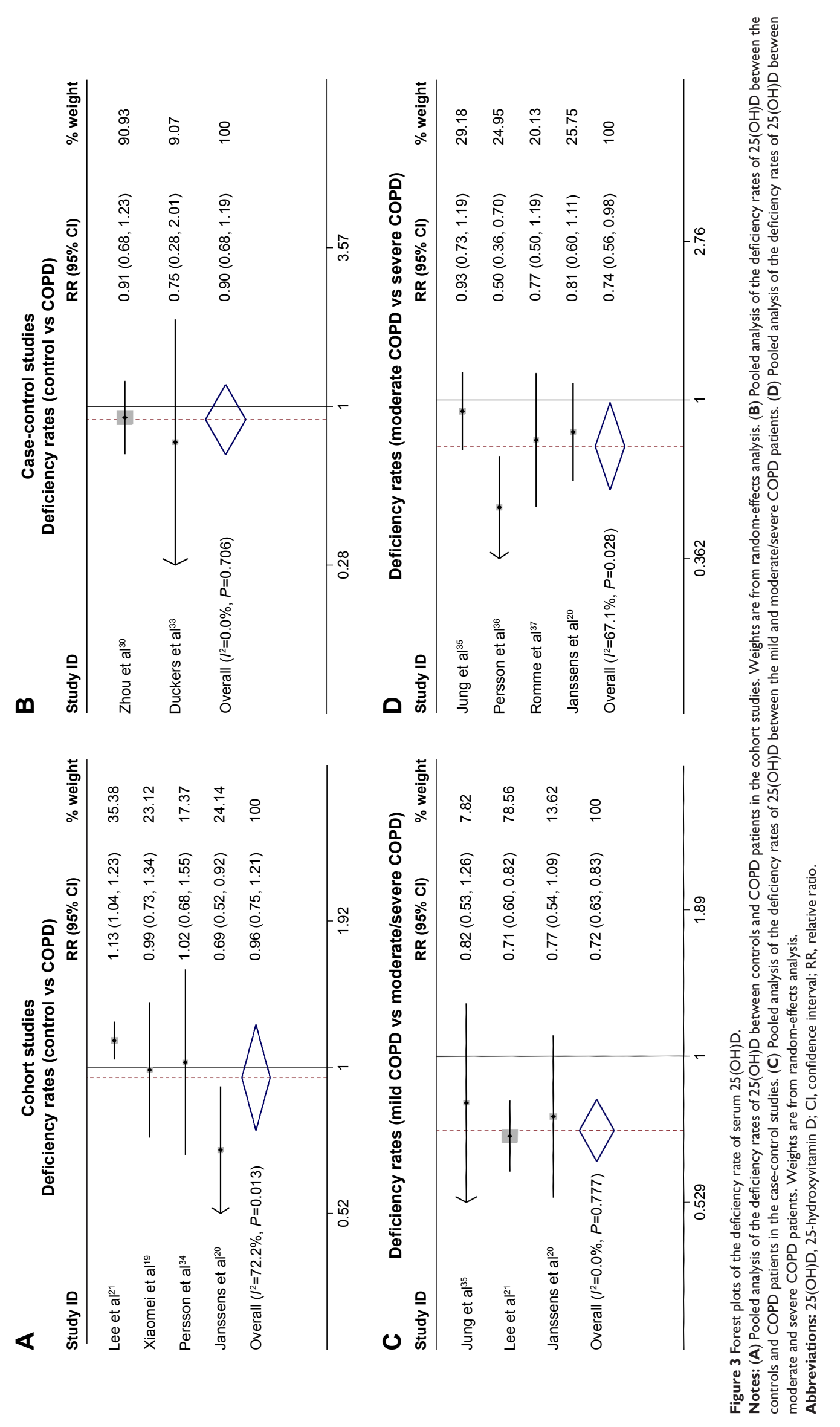


between these two groups based on a fixed-effect metaanalysis with a pooled RR of 0.899 (95\% CI: 0.678-1.192, $P=0.46$ ) (Figure 3B). In addition, no significant heterogeneity was identified with $I^{2}$ of $0(P=0.706)$. The publication bias was not be determined because only two studies were analyzed.

In addition, the deficiency rate of $25(\mathrm{OH}) \mathrm{D}$ between mild, moderate, and severe COPD patients was analyzed. A fixedeffect meta-analysis showed that the deficiency rate of $25(\mathrm{OH})$ $\mathrm{D}$ in moderate/severe COPD patients was significantly lower than that in mild COPD patients with a pooled RR of 0.723 (95\% CI: 0.632-0.828, $P<0.001$ ) (Figure 3C). No significant heterogeneity with $I^{2}$ of $0(P=0.777)$ and no publication bias was observed based on the Egger's test of $0.018(P=0.069)$. A random-effects model for meta-analysis between moderate and severe COPD patients showed the same results. ${ }^{20,35-37}$ The deficiency rate of $25(\mathrm{OH}) \mathrm{D}$ in severe COPD patients was significantly lower than that in moderate COPD patients with a pooled RR of 0.743 (95\% CI: $0.561-0.984, P=0.038)$ (Figure 3D). Significant heterogeneity was observed with $I^{2}$ of $67.1(P=0.028)$ and no publication bias was identified based on the Egger's test of $0.749(P=0.548)$.

\section{Systematic analysis of the curative effects of vitamin D supplement}

Five randomized studies were included for systematic analysis of the curative effects of vitamin D supplement in COPD patients (Table 3 ). Four studies showed that vitamin D intake benefited COPD patients. . $38,39,41$ One of the four reporters suggested that vitamin D intake inhibited COPD exacerbation among severe patients, ${ }^{41}$ and one showed that patients with vitamin D intervention had significant improvements in inspiratory muscle strength and maximal oxygen uptake. ${ }^{39}$ The other two studies demonstrated that vitamin D protected against exacerbation in patients with a baseline serum level of $25(\mathrm{OH}) \mathrm{D}$ less than $20 \mathrm{ng} / \mathrm{mL} .{ }^{9,38}$ Only the study conducted by Bjerk et al reported that there was no obvious benefit for severe COPD patients with vitamin D supplement based on two questionnaire scores (Short Physical Performance Battery and St George's Respiratory Questionnaire). ${ }^{40}$ Generally, vitamin D intake was a safe approach without severe side effects for COPD patients based on most studies.

\section{Discussion}

The critical extra-skeletal effects of vitamin D has been widely recognized in recent years, ${ }^{6}$ particularly its roles in chronic diseases such as the chronic lung disease COPD. Several studies have reported that VDBP genotype was associated with high COPD susceptibility, ${ }^{15,42,43}$ suggesting

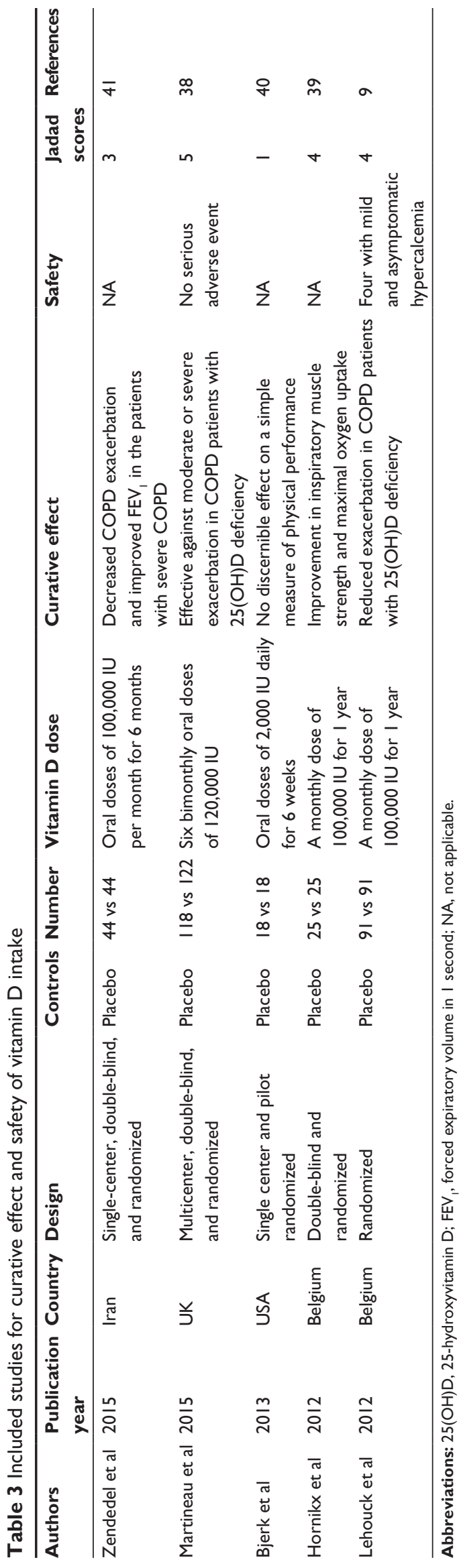


that the serum level of $25(\mathrm{OH}) \mathrm{D}$ was linked with COPD susceptibility. However, VDBP level had no effects on serum 25(OH)D concentration. ${ }^{44}$ Thus, this analysis was conducted to evaluate the association between the serum levels of $25(\mathrm{OH}) \mathrm{D}$ and the susceptibility and severity of COPD.

While the pooled results of nine studies demonstrated significant difference in the serum level of 25(OH)D between the controls and COPD patients, heterogeneity and publication bias were identified. The significant difference in the serum level of 25(OH)D between the controls and COPD patients was not identified based on the pooled analysis of cohort studies. More importantly, the deficiency rate of $25(\mathrm{OH}) \mathrm{D}$ was also comparable between these two groups based on pooled RR with cohort studies and pooled OR with case-control studies, in which much higher number of controls and patients were included. In addition, no publication bias was identified in these analyses, suggesting the high confidence of these results. While the serum level of $25(\mathrm{OH}) \mathrm{D}$ was not associated with COPD, serum deficiency of $25(\mathrm{OH}) \mathrm{D}(<20 \mathrm{ng} / \mathrm{mL})$ was significantly associated with COPD severity based on the meta-analyses between mild and moderate/severe COPD as well as moderate and severe COPD. These results suggested that the low serum level of 25(OH)D was not associated with COPD susceptibility; however, high deficiency of 25(OH)D was associated with COPD severity. Although vitamin D deficiency may be a consequence rather than the cause of $\mathrm{COPD},{ }^{45}$ the evidence was not sufficient.

It has been shown that vitamin D intake inhibited asthma exacerbations ${ }^{45}$ and accelerated clinical and radiographic improvement in tuberculosis patients. ${ }^{46}$ Our results showed that vitamin D supplement inhibited COPD exacerbation and improved inspiratory muscle strength and maximal oxygen uptake in severe COPD patients. In addition, vitamin D prevents COPD exacerbation in patients with baseline serum $25(\mathrm{OH}) \mathrm{D}$ concentrations of less than $20 \mathrm{ng} / \mathrm{mL}$. Given that serum $25(\mathrm{OH}) \mathrm{D}$ deficiency was mainly observed in severe COPD patients, the protective effects of $25(\mathrm{OH}) \mathrm{D}$ in severe COPD patients was compared with that in mild COPD patients. While our results are not consistent with the study conducted by Bjerk et a $\mathrm{a}^{40}$ our conclusion was not influenced. First, a limited number of patients $(n=36)$ were included in that study. Second, vitamin D was supplied at a dose of 2,000 IU per day and once daily for 6 weeks, which was much less than the dose used in other studies (Table 3). Several potential mechanisms could explain vitamin D deficiency in severe COPD patients. First, the evidence from epidemiological studies showed that vitamin D deficiency is a risk factor for respiratory infection. ${ }^{47,48}$ Second, it has been hypothesized that the changes of airway smooth muscle was the sole manifestation of vitamin D deficiency. ${ }^{49}$ Third, the positive association between the high level of vitamin $\mathrm{D}$ at birth and total and inhalant allergen-specific IgE may be attributable to a different mechanism. ${ }^{50}$ Thus, vitamin D supplementation may significantly alleviate COPD.

Our meta-analysis has several limitations. First, the studies exploring the relationship between 25(OH)D and COPD or COPD severity were lacking in relation to African ethnicity as only one study contained African ethnicity. ${ }^{21}$ Second, some meta-analysis results were based on random-effects model which may not be strong, and publication bias was identified in the meta-analysis. While systematic analysis of the curative effects of vitamin D supplement was analyzed on the randomized studies, the dose and treatment duration of vitamin D supplementation varied, and distinct indicators of treatment effects were used in different studies. Thus, more studies should be performed for better understanding of the role of vitamin D in COPD patients.

\section{Conclusion}

In conclusion, our results suggest that the low serum level of 25(OH)D was not associated with COPD susceptibility, but the high rate of $25(\mathrm{OH}) \mathrm{D}$ deficiency was associated with COPD severity. Vitamin D supplementation can inhibit COPD exacerbation.

\section{Author contributions}

Biyuan Zhu, Biqing Zhu, and Chaolie Xiao collected the data and did the statistical analysis. Biyuan Zhu and Biqing Zhu wrote the article. Chaolie Xiao revised the article. Zhiwen Zheng contributed to the conception and design of the study and revised the article.

\section{Disclosure}

The authors report no conflict of interests in this work.

\section{References}

1. Decramer M, Janssens W, Miravitlles M. Chronic obstructive pulmonary disease. Lancet. 2012;379:1341-1351.

2. Lim S, Lam DC, Muttalif AR, et al. Impact of chronic obstructive pulmonary disease (COPD) in the Asia-Pacific region: the EPIC Asia population-based survey. Asia Pac Fam Med. 2015;14:4.

3. Pauwels RA, Buist AS, Calverley PM, Jenkins CR, Hurd SS; GOLD Scientific Committee. Global strategy for the diagnosis, management, and prevention of chronic obstructive pulmonary disease. NHLBI/WHO Global Initiative for Chronic Obstructive Lung Disease (GOLD) Workshop summary. Am J Respir Crit Care Med. 2001;163(5):1256-1276.

4. Lopez AD, Shibuya K, Rao C, et al. Chronic obstructive pulmonary disease: current burden and future projections. Eur Respir J. 2006;27: 397-412.

5. Faner R, Gonzalez N, Cruz T, Kalko SG, Agustí A. Systemic inflammatory response to smoking in chronic obstructive pulmonary disease: evidence of a gender effect. PLoS One. 2014;9:e97491. 
6. Holick MF. Vitamin D deficiency. N Engl J Med. 2007;357:266-281.

7. Bischoff-Ferrari HA, Giovannucci E, Willett WC, Dietrich T, Dawson-Hughes B. Estimation of optimal serum concentrations of 25-hydroxyvitamin D for multiple health outcomes. Am J Clin Nutr. 2006;84:18-28.

8. Haroon M, Fitzgerald O. Vitamin D and its emerging role in immunopathology. Clin Rheumatol. 2012;31:199-202.

9. Lehouck A, Mathieu C, Carremans C, et al. High doses of vitamin D to reduce exacerbations in chronic obstructive pulmonary disease: a randomized trial. Ann Intern Med. 2012;156:105-114.

10. Garland CF, Garland FC, Gorham ED, et al. The role of vitamin D in cancer prevention. Am J Public Health. 2006;96:252-261.

11. Litonjua AA, Weiss ST. Is vitamin D deficiency to blame for the asthma epidemic? J Allergy Clin Immunol. 2007;120:1031-1035.

12. Ginde AA, Mansbach JM, Camargo CA Jr. Association between serum 25-hydroxyvitamin $\mathrm{D}$ level and upper respiratory tract infection in the Third National Health and Nutrition Examination Survey. Arch Intern Med. 2009;169:384-390.

13. Arnedo-Pena A, Juan-Cerdán JV, Romeu-García MA, et al. Vitamin D status and incidence of tuberculosis infection conversion in contacts of pulmonary tuberculosis patients: a prospective cohort study. Epidemiol Infect. 2015;143:1731-1741.

14. Pfeffer PE, Hawrylowicz CM. Vitamin D and lung disease. Thorax. 2012;67:1018-1020.

15. Horita N, Miyazawa N, Tomaru K, Inoue M, Ishigatsubo Y, Kaneko T. Vitamin D binding protein genotype variants and risk of chronic obstructive pulmonary disease: a meta-analysis. Respirology. 2015;20: 219-225.

16. Powe CE, Karumanchi SA, Thadhani R. Vitamin D-binding protein and vitamin D in blacks and whites. $N$ Engl J Med. 2014;370:880-881.

17. Powe CE, Evans MK, Wenger J, et al. Vitamin D-binding protein and vitamin D status of black Americans and white Americans. $N$ Engl J Med. 2013;369:1991-2000.

18. Jackson AS, Shrikrishna D, Kelly JL, et al. Vitamin D and skeletal muscle strength and endurance in COPD. Eur Respir J. 2013;41:309-316.

19. Xiaomei W, Hang X, Lingling L, Xuejun L. Bone metabolism status and associated risk factors in elderly patients with chronic obstructive pulmonary disease (COPD). Cell Biochem Biophys. 2014;70: 129-134.

20. Janssens W, Bouillon R, Claes B, et al. Vitamin D deficiency is highly prevalent in COPD and correlates with variants in the vitamin D-binding gene. Thorax. 2010;65:215-220.

21. Lee HM, Liu M, Lee K, Luo Y, Wong ND. Does low vitamin D amplify the association of COPD with total and cardiovascular disease mortality? Clin Cardiol. 2014;37:473-478.

22. Eagan TM, Ueland T, Wagner PD, et al. Systemic inflammatory markers in COPD: results from the Bergen COPD Cohort Study. Eur Respir J. 2010;35:540-548.

23. Donaldson GC, Seemungal TA, Bhowmik A, Wedzicha JA. Relationship between exacerbation frequency and lung function decline in chronic obstructive pulmonary disease. Thorax. 2002;57:847-852.

24. Rabe KF, Hurd S, Anzueto A, et al. Global strategy for the diagnosis, management, and prevention of chronic obstructive pulmonary disease: GOLD executive summary. Am J Respir Crit Care Med. 2007;176 532-555.

25. Fabbri LM, Hurd SS; GOLD Scientific Committee. Global Strategy for the Diagnosis, Management and Prevention of COPD: 2003 update. Eur Respir J. 2003;22:1-2.

26. Wells GA, Shea B, O Connell D, et al. The Newcastle-Ottawa Scale (NOS) for assessing the quality of nonrandomised studies in meta-analyses. In, 2000. Available from : http://www.medicine.mcgill.ca/rtamblyn/ Readings/The $\% 20$ Newcastle $\% 20-\% 20$ Scale $\% 20$ for $\% 20$ assessing $\% 20$ the $\% 20$ quality $\% 20$ of $\% 20$ nonrandomised $\% 20$ studies $\% 20$ in $\% 20$ metaanalyses.pdf. Accessed August 31, 2015.

27. Zhang H, Zhou YP, Peng HJ, et al. Predictive symptoms and signs of severe dengue disease for patients with dengue fever: a meta-analysis. Biomed Res Int. 2014;2014:359308.
28. Jadad AR, Moore RA, Carroll D, et al. Assessing the quality of reports of randomized clinical trials: is blinding necessary? Control Clin Trials. 1996;17:1-12.

29. Mahlin C, von Sydow H, Osmancevic A, et al. Vitamin D status and dietary intake in a Swedish COPD population. Clin Respir J. 2014;8: 24-32.

30. Zhou X, Han J, Song Y, Zhang J, Wang Z. Serum levels of 25hydroxyvitamin $\mathrm{D}$, oral health and chronic obstructive pulmonary disease. J Clin Periodontol. 2012;39:350-356.

31. Yang YM, Guo YF, Zhang HS, Sun TY. Antimicrobial peptide LL-37 circulating levels in chronic obstructive pulmonary disease patients with high risk of frequent exacerbations. $J$ Thorac Dis. 2015;7:740-745.

32. Yang H, Long F, Zhang $\mathrm{Y}$, et al. 1 $\alpha, 25$-Dihydroxyvitamin D3 Induces Neutrophil Apoptosis through the p38 MAPK Signaling Pathway in Chronic Obstructive Pulmonary Disease Patients. PLoS One. 2015;10: $\mathrm{e} 0120515$.

33. Duckers JM, Evans BA, Fraser WD, Stone MD, Bolton CE, Shale DJ. Low bone mineral density in men with chronic obstructive pulmonary disease. Respir Res. 2011;12:101.

34. Persson LJ, Aanerud M, Hiemstra PS, Hardie JA, Bakke PS, Eagan TM. Chronic obstructive pulmonary disease is associated with low levels of vitamin D. PLoS One. 2012;7:e38934.

35. Jung JY, Kim YS, Kim SK, et al. Relationship of vitamin D status with lung function and exercise capacity in COPD. Respirology. 2015;20(5): 782-789.

36. Persson LJ, Aanerud M, Hiemstra PS, et al. Vitamin D, vitamin D binding protein, and longitudinal outcomes in COPD. PLoS One. 2015; 10:e0121622.

37. Romme EA, Rutten EP, Smeenk FW, Spruit MA, Menheere PP, Wouters EF. Vitamin D status is associated with bone mineral density and functional exercise capacity in patients with chronic obstructive pulmonary disease. Ann Med. 2013;45:91-96.

38. Martineau AR, James WY, Hooper RL, et al. Vitamin D3 supplementation in patients with chronic obstructive pulmonary disease (ViDiCO): a multicentre, double-blind, randomised controlled trial. Lancet Respir Med. 2015;3:120-130.

39. Hornikx M, Van Remoortel H, Lehouck A, et al. Vitamin D supplementation during rehabilitation in COPD: a secondary analysis of a randomized trial. Respir Res. 2012;13:84.

40. Bjerk SM, Edgington BD, Rector TS, Kunisaki KM. Supplemental vitamin D and physical performance in COPD: a pilot randomized trial. Int J Chron Obstruct Pulmon Dis. 2013;8:97-104.

41. Zendedel A, Gholami M, Anbari K, Ghanadi K, Bachari EC, Azargon A. Effects of Vitamin D Intake on FEV and COPD Exacerbation: A Randomized Clinical Trial Study. Glob J Health Sci. 2015;7: $243-248$.

42. Xie X, Zhang Y, Ke R, et al. Vitamin D-binding protein gene polymorphisms and chronic obstructive pulmonary disease susceptibility: A meta-analysis. Biomed Rep. 2015;3:183-188.

43. Xiao M, Wang T, Zhu T, Wen F. Dual role of vitamin D-binding protein $1 \mathrm{~F}$ allele in chronic obstructive pulmonary disease susceptibility: a meta-analysis. Genet Mol Res. 2015;14:3534-3540.

44. Ponda MP, McGee D, Breslow JL. Vitamin D-binding protein levels do not influence the effect of vitamin D repletion on serum PTH and calcium: data from a randomized, controlled trial. J Clin Endocrinol Metab. 2014;99:2494-2499.

45. Foong RE, Zosky GR. Vitamin D deficiency and the lung: disease initiator or disease modifier? Nutrients. 2013;5:2880-2900.

46. Salahuddin N, Ali F, Hasan Z, Rao N, Aqeel M, Mahmood F. Vitamin D accelerates clinical recovery from tuberculosis: results of the SUCCINCT Study [Supplementary Cholecalciferol in recovery from tuberculosis]. A randomized, placebo-controlled, clinical trial of vitamin D supplementation in patients with pulmonary tuberculosis'. Bmc Infect Dis. 2013;13:22.

47. Urashima M, Segawa T, Okazaki M, Kurihara M, Wada Y, Ida H. Randomized trial of vitamin D supplementation to prevent seasonal influenza A in schoolchildren. Am J Clin Nutr. 2010;91:1255-1260. 
48. Camargo CA Jr, Ingham T, Wickens K, et al. Cord-blood 25hydroxyvitamin D levels and risk of respiratory infection, wheezing, and asthma. Pediatrics. 2011;127:e180-e187.

49. Gupta A, Sjoukes A, Richards D, et al. Relationship between serum vitamin $\mathrm{D}$, disease severity, and airway remodeling in children with asthma. Am J Respir Crit Care Med. 2011;184:1342-1349.
50. Wittke A, Weaver V, Mahon BD, August A, Cantorna MT. Vitamin D receptor-deficient mice fail to develop experimental allergic asthma. J Immunol. 2004;173:3432-3436.

\section{Publish your work in this journal}

The International Journal of COPD is an international, peer-reviewed journal of therapeutics and pharmacology focusing on concise rapid reporting of clinical studies and reviews in COPD. Special focus is given to the pathophysiological processes underlying the disease, intervention programs, patient focused education, and self management protocols.
This journal is indexed on PubMed Central, MedLine and CAS. The manuscript management system is completely online and includes a very quick and fair peer-review system, which is all easy to use. Visit http://www.dovepress.com/testimonials.php to read real quotes from published authors. 